JOCHEN FRANZKE

Universität Potsdam, Potsdam

DOI : $10.14746 /$ rie.2017.11.11

\title{
Ansätze künftiger deutscher Russlandpolitik in Wahlprogrammen von sechs politischen Parteien zur Bundestagswahl 2017
}

Wahlprogramme sind in Deutschland wie in anderen demokratischen Staaten strategische politische Texte, in welchen wesentliche inhaltliche Ziele einer politischen Partei formuliert sind. Die betreffende Partei verpflichtet sich mit diesen gegenüber ihren potentiellen Wählern im Falle eines Wahlsieges diese Ziele in der kommenden Legislaturperiode umzusetzen.

Wahlprogramme konkretisieren die zumeist allgemein formulierten Inhalte längerfristig konzipierter Grundsatzprogramme der betreffenden politischen Parteien (Siehe Korte, 2013). Diese Dokumente können als Kondensat der langjährigen Diskussionen innerhalb einer Partei aufgefasst werden. Sie erlauben Rückschlüsse auf die innerparteilichen Prozesse und die dabei erzielten Kompromisse bzw. die gesetzten politischen Prioritäten. Wahlprogramme werden im Allgemeinen ohne Rücksicht auf mögliche Koalitionspartner einer späteren Regierung beschlossen und spiegeln die Parteiprogrammatik weitgehend „pur“ dar.

Wahlprogramme dienen verschiedenen Zwecken. Sie haben nach innen und außen gerichtete Funktionen. Nach Innen sollen diese die Identifikation der Parteimitglieder für die Partei stärken und diese für den bevorstehenden Wahlkampf mobilisieren. Nach Außen sollen sie die eigene Stammwählerschaft mobilisieren und für die politischen Ziele bei den anderen Wählern bzw. den Nichtwählern werben (Siehe Kercher, Brettschneider, 2013). Sie dienen auch als Mittel zu deren Profilierung gegenüber anderen Mitbewerbern und vor allem als Instrument im Wahlkampf. Nahezu alle Parteien konzentrieren sich in ihrer direkten Wählerkommunikation auf zentrale Themen ihrer Wahlprogramme (Siehe Römmele, 2013).

$\mathrm{Da}$ in Deutschland traditionell nach Wahlen fast immer Koalitionsregierungen gebildet werden, dienen die Wahlprogramme (gemeinsam mit den Grundsatzprogrammen) auch als Ausgangspunkt bzw. Leitlinie der Koalitionsverhandlungen und der dabei einzugehenden Kompromisse. Unter diesen Umständen können eine Reihe von Inhalten der betreffenden Wahlprogramme nicht umgesetzt werden. Unter Berücksichtigung dieser Tatsache ist dennoch nachweisbar, dass sich „das parlamentarische Handeln nach der Wahl durchaus stark mit den Absichtserklärungen der Wahlprogramme deckt" (Westle et al., 2014, S. 320; siehe auch Hofferbert, Klingemann, 1990; Rölle, 2000).

In diesem Beitrag werden die Wahlprogramme von sechs Parteien (CDU/CSU, SPD, Linke, Bündnis90/Die Grünen, FDP sowie AfD) für die Bundestagswahl am 24. September 2017 in Bezug auf deren Aussagen zur künftigen deutschen bzw. europä- 
ischen Politik gegenüber Russland ausgewertet. ${ }^{1}$ Diese politischen Parteien wurden ausgewählt, da sie eine realistische Chance auf den Einzug in den 19. Deutschen Bundestag haben.

Jeder der genannten Parteien hat natürlich eine dezidierte Position zur künftigen deutschen und europäischen Politik gegenüber Russland. Dies belegt eine Vielzahl von Dokumenten der laufenden Legislaturperiode, die in diesem Beitrag nicht weiter zitiert werden sollen. Ob die genannten Positionen auch in den jeweiligen Wahlprogrammen auftauchen hängt $u$. a. von drei Faktoren ab. Erstens sind die vorliegenden Wahlprogramme unterschiedlich detailliert. ${ }^{2}$ Zweitens sind außenpolitische Prioritäten in diesen Wahlprogrammen traditionell nachrangig gegenüber innenpolitischen Prioritäten. Drittens ist die internationale Lage augenblicklich von einer außergewöhnlichen Vielzahl von internationalen Konflikten und große Unsicherheiten geprägt. Alle drei Gründe können dazu führen, dass die Position der jeweiligen Partei zu Russland im jeweiligen Wahlprogramm nur verkürzt dargestellt oder gar nicht erwähnt wird.

\section{Aussagen der Wahlprogramme 2017 zur Russlandpolitik}

Im folgenden Kapitel sollen erst einmal die Aussagen der Parteien zum Thema ohne weitere Kommentierung zitiert werden. Hintergrund der Aussagen der Wahlprogramme zur Russlandpolitik ist die dramatische Verschlechterung der Beziehungen Deutschlands und der EU zu Russland nach der Annexion der Krim im Frühjahr 2014 und die anhaltende Infragestellung der territorialen Integrität Ukraine durch russische Aggression in der Ost-Ukraine. Diese hatte zum „Ende der strategischen Partnerschaft“ geführt (Franzke, 2016, S. 107). Mittlerweise sind weitere Konfliktfelder hinzugekommen, wie das russische Eingreifen in den Syrienkrieg an der Seite des Assad-Regime, die von Moskau ausgehenden Cyber-War-Aktivitäten sowie dessen Versuche, sich in den Wahlkämpfe in demokratischen Staaten, draunter in Deutschland, direkt einzumischen.

\section{Union}

Die künftige Russlandpolitik spielt im Wahlprogramm der CDU/CSU ${ }^{3}$ keine besondere Rolle (Siehe CDU/CSU, 2017). Dies ist möglicherweise auf unterschiedliche Positionen zurückzuführen, da die bayrische CSU seit einiger Zeit eine deutlich prorussische Politik betreibt als die CDU und deren Kanzlerin Angela Merkel.

Nur an zwei Stellen wird Russland in diesem Wahlprogramm überhaupt nur kurz erwähnt. Erstens wird darauf verwiesen, dass ,,selbst in unserer Nachbarschaft ... die

${ }^{1}$ Die Wahlprogramme wurden von Bundesparteitagen beschlossen und besitzen daher eine hohe Verbindlichkeit. Die geschah am 17. Mai 2017 (FDP) sowie 18. Mai 2017 (AfD), 23. Mai 2017 (SPD), 11. Juni 2017 (Die Linke), 18. Juni 2017 (Bündnis90/Grüne) und 3. Juli 2017 (CDU/CSU).

2 Deren Umfang reicht von 76 Seiten (AfD), über 78 Seiten (Union), 88 Seiten (SPD), 95 Seiten (FDP), 106 (Bündnis 90/Die Grünen) bis zu 136 Seiten (Die Linken).

${ }^{3}$ Die CSU will mit dem Bayernplan 2017-2021 ein eigenes Wahlprogramm für die Bundestagswahl am 23. Juli 2017 verabschieden. 
territoriale Integrität der Ukraine durch die russische Aggression in Frage gestellt (ist)“ (CDU/CSU, 2017, S. 55). Zweitens spricht das Programm vom EU-Engagement ,im Russland-Ukraine-Konflikt..."(S. 56).

Deutlich wird, dass die CDU/CSU auf europäische Lösungen setzt, die gilt offenbar auch für das Verhältnis zu Russland. Im Programm heißt es dazu: „Wir müssen unsere gemeinsame geostrategische Verantwortung für Freiheit und Frieden wahrnehmen und bei der Bewältigung von Konflikten in unserer Nachbarschaft mithelfen" (CDU/CSU, 2017, S. 56). Die EU wird dabei als Wertegemeinschaft verstanden, die dem Schutz von Demokratie, Rechtsstaatlichkeit und Religionsfreiheit verpflichtet ist. „An diesen Kriterien mussten sich alle Länder messen lassen, die bisher der EU beigetreten sind“ (CDU/CSU, 2017, S. 57).

\section{SPD}

Das Wahlprogramm der SPD (Siehe SPD, 2017) widmet sich dem Verhältnis zu Russland hingegen ausführlicher. In einen eigenen Absatz heißt es: „Unser Verhältnis zu Russland ist durch das Vorgehen der russischen Regierung in der Ostukraine und den Bruch des Völkerrechts durch die Annexion der Krim belastet. Fundamentale Prinzipien der europäischen Friedens- und Sicherheitsordnung sind verletzt. Wir sind jedoch davon überzeugt, dass Frieden und Sicherheit in Europa nur mit, nicht ohne oder gar gegen Russland möglich sind. Dafür bedarf es der Deeskalation, der Rückkehr zum politischen Dialog und der differenzierten Anwendung von Sanktionsmechanismen. Der politische Prozess für die Ostukraine, der den Ausbruch eines offenen Krieges erfolgreich verhindern konnte, ist ins Stocken geraten. Wir halten dennoch an ihm fest. Substanzielle Fortschritte bei der Umsetzung des Minsker Abkommens würden eine schrittweise Aufhebung der Sanktionen gegen Russland nach sich ziehen. Wir streben langfristig eine inklusive Sicherheitsarchitektur für ganz Europa an“" (SPD, 2017, S. 84).

Auffallend ist, dass im SPD-Wahlprogramm das Verhältnis zu Russland vielfältig institutionell verankert ist. Es wird z. B. als „Herausforderung“ angesehen, bei der ,es auf eine handlungsfähige transatlantische Partnerschaft ankommt" (SPD, 2017, S. 84). Dies gelte auch für den Europarat und die OSZE.

Die SPD ordnet ihre Vorstellungen einer künftigen Russlandpolitik in ihren grundlegenden Anspruch als „Friedenspartei (ein), die ... für eine Außenpolitik der klugen Diplomatie (steht). Im Geiste der Entspannungspolitik Willy Brandts ist es unser Anspruch, Brücken zu bauen. Aufrüstung und Säbelrasseln lösen keine Konflikte. ${ }^{4}$ Euro-

${ }^{4}$ Hier bezieht sich das Wahlprogramm eindeutig auf eine Position des damaligen deutschen Außenministers Frank-Walter Steinmeier (SPD), der am 18.6.2016 in einem Interview mit „BILD am SONNTAG“ folgendes: „Was wir jetzt nicht tun sollten, ist durch lautes Säbelrasseln und Kriegsgeheul die Lage weiter anzuheizen. Wer glaubt, mit symbolischen Panzerparaden an der Ostgrenze des Bündnisses mehr Sicherheit zu schaffen, der irrt. Wir sind gut beraten, keine Vorwände für eine neue, alte Konfrontation frei Haus zu liefern... Es wäre fatal, jetzt den Blick auf das Militärische zu verengen und allein in einer Abschreckungspolitik das Heil zu suchen“" (BamS, 18.6.2016). Diese Bemerkungen beziehen sich auf NATO-Manöver, die zu diesem Zeitpunkt im Baltikum und in Polen stattfanden. 
pa, die USA, Russland und China tragen gemeinsam Verantwortung für den Frieden in der Welt und den Kampf gegen den internationalen Terrorismus. Deshalb können nur der Dialog zwischen den Regierungen, die Verständigung zwischen den Völkern und eine gelebte Solidarität zwischen Nord und Süd zu mehr globaler Sicherheit führen“ (SPD, 2017, S. 4).

\section{Linke}

Die Linke nimmt in ihrem Wahlprogramm ausführlich zum Verhältnis zu Russland Stellung (Siehe Linke, 2017). ${ }^{5}$ Dazu heißt es: „Die NATO dehnt ihren Einflussbereich bis an die Westgrenze Russlands aus. Der Beitritt weiterer Länder zur NATO wie Georgien, der Ukraine oder Mazedonien würde die Spannungen mit Russland weiter anheizen. Viele Menschen in unserem Land sind beunruhigt wegen der Verschlechterung der Beziehungen Deutschlands und der EU zu Russland. Von einem gemeinsamen Haus Europa, von der Friedensdividende nach dem Ende des Kalten Krieges, spricht heute keiner mehr. Stattdessen findet mitten in Europa, in der Ukraine, ein heißer Krieg statt. Zwischen der EU und Russland bestimmen Sanktionen und Gegensanktionen das Bild. Wo Abrüstung geboten wäre, dominiert auf beiden Seiten verbale und militärische Aufrüstung. Diplomatie und militärische Zurückhaltung sind ins Abseits geraten. Wir halten diese Konfrontationspolitik für fatal“" (Linke, 2017, S. 106).

Die Linke ordnet die Russlandpolitik in einen größeren institutionellen und inhaltlichen Rahmen ein. Dazu wird festgestellt: „DIE LINKE tritt für die Stärkung der OSZE als wichtigem Format der gesamteuropäischen Kooperation ein. Um dies zu erreichen, muss als Erstes eine Vereinbarung getroffen werden, keine neuen Mitglieder in die NATO aufzunehmen. in die NATO aufzunehmen. Wir wollen eine neue, auf Entspannung orientierte Ostpolitik. Sicherheit in Europa kann nur Sicherheit mit und nicht gegen Russland sein. Das muss die sicherheitspolitische Orientierung von europäischem und deutschem Handel werden. Militärmanöver oder Pläne zur Stationierung von Waffensystemen entlang der russischen Westgrenze heizen Konflikte an. Wir setzen uns gegen Militärmanöver ein. Den Einsatz und die Stationierung deutscher Soldaten in den osteuropäischen Staaten wollen wir unterbinden. Das NATO-Rüstungsziel von zwei Prozent des BIP lehnen wir ab. Wir wollen die NATO auflösen und durch ein kollektives Sicherheitssystem unter Einbeziehung von Russland ersetzen, das auf Abrüstung zielt. Wir wollen, dass die Bundeswehr dem Oberkommando der NATO entzogen wird und die Bundesrepublik aus den militärischen Strukturen des Bündnisses austritt... Wir wollen die US- und NATO-Infrastruktur in Deutschland beseitigen, die für den Aufmarsch gegen Russland, eine verheerende Regime Change-Politik sowie ganz allgemein für Interventionskriege genutzt wird“" (Linke, 2017, S. 106).

${ }^{5}$ Innerhalb der Partei gibt es allerdings auch einen russlandkritischen gemäßigten Reformerflügel. Dessen Anträge fand allerdings keine Mehrheit der Delegierten des Bundesparteitages, der über das Wahlprogramm abgestimmte. Die Antragen sahen eine Verurteilung der Krim-Annexion als völkerrechtswidrig und eine Verurteilung des Bürgerkrieges in der Ost-Ukraine vor. Auch die Verurteilung von Menschenrechtsverletzungen in Russland und China schaffte es nicht ins Wahlprogramm. (Der Tagesspiegel, 12.6.2017). 


\section{Bündnis 90/Die Grünen}

Bündnis 90/Die Grünen nehmen in ihrem Wahlprogramm nur knapp, aber sehr präzise zur künftigen Russlandpolitik Deutschlands Stellung (Siehe Bündnis90/Die Grünen, 2017). Im Wahlprogramm der Grünen heißt es dazu: „Die zunehmend aggressive Großmachtpolitik von Präsident Putin hat Russland zu einer Bedrohung der europäischen Friedensordnung gemacht. In der Ukraine, dem Nachbarland der EU, wird Krieg geführt, seitdem Russland Teile des Landes völkerrechtswidrig annektiert hat. Infolgedessen haben die Spannungen zwischen Russland und dem NATO-Bündnis die längst überwunden geglaubte Abschreckungslogik wieder in Gang gesetzt" (Bündnis90/Die Grünen, 2017, S. 31).

Die Grünen verweisen auch auf die internationale Dimension russischer Politik. In ihrem Wahlprogramm wird festgestellt: „Der syrische Bürgerkrieg hat sich zu einem überaus grausamen regionalen Stellvertreterkrieg entwickelt, in dem Russland an der Seite des Assad-Regimes mit brutalem militärischem Eingreifen und der menschenverachtenden Bombardierung von Zivilisten Fakten schafft" (Bündnis90/Die Grünen, 2017, S. 31).

Institutionell setzen die Grünen vor allem auf die EU und deren gemeinsame Außen- und Sicherheitspolitik. ,Wir Grünen setzen uns für eine stärkere Europäisierung der Außen-, Entwicklungs- und Sicherheitspolitik ein. Kein europäisches Land ist allein in der Lage, den internationalen Herausforderungen erfolgreich zu begegnen. Das gilt umso mehr in einer Zeit, in der sich durch die aggressive Großmachtpolitik Russlands unter Präsident Putin, die von Abschottung und nationalistischem Denken geprägte Politik des amerikanischen Präsidenten Trump und die vielen Krisenherde im Nahen Osten und in Nordafrika die Rahmenbedingungen für die Sicherheit der EU grundlegend ändern“" (Bündnis90/Die Grünen, 2017, S. 35).

\section{Freie Demokraten}

Im liberalen Wahlprogramm findet sich ein eigener Abschnitt unter der Überschrift „Klare Haltung gegenüber Russland“ (Siehe FDP, 2017). Darin heißt es: „Wir Freie Demokraten fordern die russische Regierung auf, die völkerrechtswidrige Besetzung der Krim und den Krieg in der Ostukraine unverzüglich zu beenden. Diese verletzen nicht nur das Gewaltverbot nach Art. 2 Ziffer 4 der Charta der Vereinten Nationen, sondern auch die Souveränität und territoriale Integrität der Ukraine. Zudem beobachten wir die zunehmende Unterdrückung der Opposition und Zivilgesellschaft in Russland mit großer Sorge und treten dafür ein, diese Menschenrechtsverletzungen klar zu benennen und zu verurteilen. Für uns gelten die Prinzipien der europäischen Friedensordnung, zu denen sich auch Russland bekannt hat: Die souveräne Gleichheit der Staaten, die Unverletzlichkeit ihrer Grenzen, die friedliche Beilegung von Konflikten sowie die freie Bündniswahl, Demokratie und Achtung der Menschenrechte. Für uns Freie Demokraten sind diese Prinzipien nicht verhandelbar“" (FDP, 2017, S. 54f.). 
Zum Sanktionsregime stellen die Liberalen fest: „Solange Präsident Putin seine Interventionspolitik fortsetzt, müssen daher die Sanktionen gegen Russland aufrechterhalten und eine Wiederaufnahme Russlands in die G8 ausgeschlossen werden. Im Falle einer erneuten militärischen Eskalation müssen die Sanktionen gegen Russland weiter verschärft werden. Im Gegenzug können Sanktionen bei einem substanziellen Einlenken der russischen Regierung gelockert oder aufgehoben werden" (FDP, 2017, S. 54ff.).

Abschließend wird festgehalten: „Als Nachbarn auf dem europäischen Kontinent sind Deutschland und die EU mit Russland eng verbunden - wirtschaftlich, kulturell und politisch. Gerade in schwierigen Zeiten ist es unerlässlich, miteinander im Gespräch zu bleiben. Ob auf politischer Ebene, wie in der OSZE oder im NATORussland-Rat, oder durch zivilgesellschaftliche Kontakte: Dialog schafft Vertrauen, Vertrauen schafft Sicherheit. Mittelfristig muss es unser Ziel sein, über Dialog und vertrauensbildende Maßnahmen wieder zu einer verlässlichen Partnerschaft mit Russland zu kommen" (FDP, 2017, S. 54f.).

Ausdrücklich stellen die Liberalen eine Verbindung zwischen der künftigen Russlandpolitik und der NATO her. Wir betrachten die NATO ,auch in Zukunft als Garant für unsere Sicherheit. Das bedeutet, dass wir die jüngsten Beschlüsse der Allianz von Wales und Warschau in vollem Umfange mittragen: Stärkung des Abschreckungs- und Verteidigungspositivs der NATO, einhergehend mit gleichzeitigem Dialog mit Russland“" (FDP, 2017, S. 55).

\section{Allianz für Deutschland}

Im kurzen Wahlprogramm der AfD heißt es: „Eine Entspannung im Verhältnis zu Russland ist für die AfD Voraussetzung für einen dauerhaften Frieden in Europa. Es liegt im deutschen Interesse, Russland in eine sicherheitspolitische Gesamtstruktur einzubinden, ohne eigene Interessen und die unserer Bündnispartner außer Acht zu lassen. Die AfD tritt für die Beendigung der Sanktionspolitik ein. Die wirtschaftliche Zusammenarbeit mit Russland sollte vielmehr vertieft werden“ (AfD, 2017, S. 18).

Des Weiteren wird festgestellt: „Die AfD setzt sich dafür ein, dass die Rolle der OSZE bei der Stabilisierung von Krisenregionen in Europa und seiner Peripherie ausgebaut wird“" (AfD, 2017, S. 18).

\section{Vergleich}

Die kritischste Sicht auf die aktuellen und künftigen Beziehungen zu Russland findet sich im Wahlprogramm der Liberalen. Hier wird die russische Regierung direkt aufgefordert, die völkerrechtswidrige Besetzung der Krim und den Krieg in der Ostukraine unverzüglich zu beenden. Außerdem ist das liberale Wahlprogramm das einzige, welches die anti-demokratische innenpolitischen Entwicklungen in Russland kritisiert. Für die anderen Parteien spielen diese mittlerweile - im Gegensatz zu der Zeit vor 2014 - offenbar keine Rolle mehr. 
Die unkritischste Sicht auf die Beziehungen zu Russland enthält das Wahlprogramm der Linken. Kritik an der russischen Politik in der Ukraine wird bis auf eine Ausnahme nicht geübt (,,auf beiden Seiten dominiere verbale und militärische Aufrüstung“'). Die Linke fordert eine Vielzahl konkreter einseitiger Vorleistungen Deutschlands, der EU bzw. der NATO, um Russland entgegenzukommen: Auf Militärmanöver oder die Stationierung von Waffensystemen entlang der russischen Westgrenze soll verzichten werden, auf die Aufnahme weiterer Mitglieder aus Osteuropa in die NATO soll ebenfalls verzichten werden und die US- und NATO-Infrastruktur in Deutschland, die für den Aufmarsch gegen Russland genutzt wird, soll beseitigt werden.

Insgesamt lassen sich Gemeinsamkeiten und Unterschieden der Wahlprogramme zur künftigen Russlandpolitik Deutschlands und der EU in einigen Thesen zusammenfassen:

1. Bezüglich der Ursachen für die Verschlechterung der Beziehungen Deutschlands und der EU zu Russland seit 2014 sind sich vier der sechs Parteien einig (Union, SPD, Liberale und Grüne). Diese liege am Vorgehen der russischen Regierung in Ostukraine und dem Bruch des Völkerrechts durch Annexion der Krim. Damit wurden fundamentale Prinzipien der europäischen Friedens- und Sicherheitsordnung verletzt. Die Linke sieht hingegen die Ursache für die Zuspitzung der Situation ausschließlich in der Politik der Institutionen des Westens, insbesondere wegen der Aufnahme neuer NATO-Mitglieder. Die Annexion der Krim wird nicht erwähnt. Die AfD geht auf die Ursachen der Krise in den deutsch-russischen Beziehungen in ihrem Wahlprogramm nicht ein.

2. Die Bereitschaft zum Dialog mit der Regierung Russlands enthalten alle sechs Wahlprogramme. Hier besteht Einigkeit, dass es angesichts der geopolitischen Lage für Deutschland dazu keine Alternative gibt. Unterschiedlich ist allerdings die Konditionalisierung des angestrebten Dialoges. Die Liberalen stellen Vorbedingungen an die russische Seite, um zu einer gemeinsamen europäischen Friedensordnung zurückzukehren. Die Linken fordern einseitige Vorleistungen Deutschlands, der EU und der NATO (siehe oben). Die anderen Parteien bleiben hier unverbindlich.

3. Abschreckung als Teil einer Doppelstrategie (Abschreckung und Dialog) gegenüber Russland wird überraschenderweise nur im Wahlprogramm der Liberalen erwähnt, die sich für eine ,Stärkung des Abschreckungs- und Verteidigungspositivs der NATO" einsetzen.

4. Die AfD spricht sich in ihrem Wahlprogramm als einzige Partei für die (offenbar bedingungslose) Beendigung der Sanktionen gegen Russland aus. Die klarste Position für ein differenzierteres Sanktionsregime findet sich im Wahlprogramm der Liberalen. Dort werden drei verschiedene Vorgehensweise vorgeschlagen: Bei Fortsetzung der russischen Interventionspolitik in der Ukraine sollen die Sanktionen aufrechterhalten, bei Eskalation verschärft sowie bei substanziellen Einlenken der russischen Regierung gelockert oder aufgehoben werden. Die SPD fordert allgemeiner eine ,differenzierte Anwendung von Sanktionsmechanismen“, nennt aber bezeichnenderweise nur die Möglichkeit der schrittweisen Aufhebung der Sanktionen. Union, Grüne und Linke äußern sich zur Zukunft der Sanktionen in ihren Wahlprogrammen nicht. 
5. Die Frage, welche multilateralen Institutionen welche Rolle in der künftigen deutschen Russlandpolitik spielen sollen, wird unterschiedlich beantwortet. Die Rolle der EU bei der künftigen Russlandpolitik wird vor allem von der Union („Gemeinsame geostrategische Verantwortung der EU für Freiheit und Frieden“) sowie den Grünen (,Stärkere Europäisierung der Außen- und Sicherheitspolitik“) hervorgehoben. Für eine starke Rolle der OSZE plädieren die Wahlprogramme der SPD, der Linken, der Grünen und der AfD. Die Rolle der NATO (NATO-Russland-Rat) heben die SPD und die Liberalen hervor. Die SPD erwähnt schließlich noch den Europarat.

\section{Szenarien künftiger deutscher Russlandpolitik}

Fasst am die Einschätzungen dieses Beitrages zusammen, so können drei Szenarien für die künftigen deutsch-russischen Beziehungen beschrieben werden. Die AfD kann dabei ausgeklammert werden, da diese keine Regierungsoption hat.

1. Bleibt die aggressive Politik des Kremls gegenüber der Ukraine unverändert, würde eine künftige Bundesregierung aus Union und SPD (unter Führung der Union) die bisherige deutsche Politik aus Dialogbereitschaft und Abschreckung fortsetzen (Status Quo Szenario). Bei einer Regierung der Union mit den Grünen bzw. den Liberalen ist eher eine härtere Haltung Deutschlands gegenüber dem Kreml zu erwarten. Eine „Große Koalition“ unter Führung der SPD könnte angesichts der russlandfreundlichen Positionen der CSU weniger stark auf Abschreckung und mehr auf Entgegenkommen gegenüber dem Kreml setzen. Der alternative Ansatz der Linken hat m. E. keine Chance auf Realisierung, weil in einer potentiell möglichen rot-rot-grünen Regierung weder die Grünen noch die SPD einer Auflösung der NATO zustimmen würden. Sollte es dennoch zu einer solchen Regierung kommen (wenn die Linke auf diese Forderung verzichtet oder deren Umsetzung auf den Sankt-Nimmerlein-Tag vertagt wird), wäre allerdings eine deutlich größere Dialogund Kompromissbereitschaft gegenüber Russland zu erwarten.

2. Für das angesichts der Erfahrungen der letzten Jahre nicht auszuschließende „pessimistische“ Szenario einer Eskalation der russischen Aggression in der Ukraine oder eines anderweitigen Konflikts zwischen Deutschland bzw. der EU und Russland finden sich in den Wahlprogrammen verständlicherweise keine Aussagen. Nur die Liberalen scheinen auf ein solches Szenario vorbereitet zu sein.

3. Das „optimistische“ Szenario geht davon hingegen aus, dass es aus aktueller Sicht Ende 2017/Anfang 2018 ein Window of Opportunity gibt, um einen „Neustart“ der deutsch-russischen bzw. EU-Russland-Beziehungen zu ermöglichen. Dies wird im wesentlichen Maße von der Kompromissbereitschaft des Kremls vor den russischen Präsidentschaftswahlen 2018, der Leistungsfähigkeit des französisch-deutschen Tandems in der EU sowie der Konstruktivität der Trump-Administration abhängen. Ob dieser Neustart letztlich „nur“ für besseres Krisenmanagement genutzt wird oder der Weg zu einer neuen Sicherheitsarchitektur für ganz Europa geebnet wird, ist heute nicht vorhersagbar. 


\section{Wahlprogramme}

AfD (2017), Programm für Deutschland. Wahlprogramm der Alternative für Deutschland für die Wahl zum Deutschen Bundestag am 24. September 2017, https://www.afd.de/wp-content/ uploads/sites/111/2017/06/2017-06-01_AfD-Bundestagswahlprogramm_Onlinefassung.pdf.

Bündnis90/Die Grünen (2017), Zukunft wird aus Mut gemacht. Entwurf des Wahlprogramms 2017, https://www.gruene.de/fileadmin/user_upload/Dokumente/Gruener_Bundestagswahlprogrammentwurf_2017.pdf.

CDU/CSU (2017), Regierungsprogramm 2017-2021. Für ein Deutschland, in dem wir gut und gerne leben, https://www.cdu.de/system/tdf/media/dokumente/170703regierungsprogramm2017. pdf?file $=1$.

SPD (2017), Es ist Zeit für mehr Gerechtigkeit: Zukunft sichern, Europa stärken. Das Regierungsprogramm 2017 bis 2021, https:/www.spd.de/fileadmin/Dokumente/Bundesparteitag_2017/ Es_ist_Zeit_fuer_mehr_Gerechtigkeit-Unser_Regierungsprogramm.pdf.

Die Linke, Die Zukunft, für die wir kämpfen: SOZIAL. GERECHT. FRIEDEN. FÜR ALLE, https://www.die-linke.de/fileadmin/download/wahlen2017/wahlprogramm2017/wahlprogramm2017.pdf.

FDP, Schauen wir nicht länger zu. Programm der Freien Demokraten zur Bundestagswahl 2017, https://www.fdp.de/sites/default/files/uploads/2017/05/29/20170523-schauen-wir-nichtlanger-zu.pdf.

\section{Bibliographie}

Franzke J. (2016), Nach dem Ende der strategischen Partnerschaft. Deutschland auf der Suche nach einer neuen Russlandpolitik, „Krakowskie Studia Międzynarodowe“ (Krakauer Internationale Studien), 2016/2, S. 107-122.

Hemmelmann P. (2017), Der Kompass der CDU: Analyse der Grundsatz- und Wahlprogramme von Adenauer bis Merkel, Springer Fachmedien, Wiesbaden.

Hofferbert R. I., Klingemann H.-D. (1990), The Policy Impact of Party Programmes and Government Declarations in the Federal Republic of Germany, „European Journal of Political Research", 18, S. 277-304.

Kercher J., Brettschneider F. (2013), Wahlprogramme als Pflichtübung? Typen, Funktionen und Verständlichkeit der Bundestagswahlprogramme 1994-2009, in: Wahlen und Wähler: Analysen aus Anlass der Bundestagswahl 2009, Herausgeber: B. Weßels, H. Schoen, O. Gabriel Hrsg., Springer VS, Wiesbaden.

Korte K.-R. (Hrsg.), Die Bundestagswahl 2013: Analysen der Wahl-, Parteien-, Kommunikationsund Regierungsforschung, Springer Verlag, Wiesbaden.

Rölle D. (2000), Wahlprogramme: Richtschnur parlamentarischen Handelns, „Zeitschrift für Parlamentsfragen“, Jg. 31, Nr. 4, S. 821-832.

Römmele A. (2013), Direkte Kommunikation zwischen Parteien und Wählern: Professionalisierte Wahlkampftechnologien in den USA und in der BRD, Springer Verlag, Wiesbaden.

Westle B., Begemann Ch., Rütter A. (2014), Wahlprogrammatik und politische Berichterstattung: Vermittlung politischer Themen und Issues durch Tageszeitungen, in: Die Bundestagswahl 2013: Analysen der Wahl-, Parteien-, Kommunikations- und Regierungsforschung, Hrsg. K.-R. Korte, Springer Verlag, Wiesbaden, S. 317-342. 


\title{
Approaches for future policy of Germany towards Russia in election programs for the 2017 Bundestag election of six German political parties
}

\begin{abstract}
Summary
In this article the electoral programs for the Bundestag elections on 24 September 2017 of six German political parties (CDU/CSU, SPD, Left Party, Bündnis90/Die Grünen, FDP, and AfD) will be evaluated for their statements on the future German and European policy on Russia. These political parties were selected because they have a realistic chance of being elected into the 19th German Bundestag. The most critical view on the relations with Russia is to be found in the liberal election program. Here, the Russian government is directly called upon to stop immediately the unlawful occupation of the Crimea and the war in the Eastern Ukraine. The most uncritical view on relations with Russia is to be found the election program of the Left party. Criticism of Russian policy in Ukraine is not practiced. However, it calls for a large number of unilateral advance steps by Germany, the EU and NATO, to meet Russia. The willingness to dialogue with the government of Russia contains all six electoral programs under evaluation. Elements of deterrence only contains the program of the FDP. Three scenarios for Germany's future relations with Russia are possible.
\end{abstract}

Key words: Germany, Russia, European Union, Political parties, Foreign policy

\section{Założenia przyszłej polityki Niemiec wobec Rosji w programach wyborczych sześciu partii politycznych w wyborach do Bundestagu w 2017 r.}

\section{Streszczenie}

W artykule autor dokonuje ewaluacji programów sześciu partii politycznych w Niemczech (CDU/CSU,SPD,Lewica,Sojusz 90/Zieloni, FDP, Alernatywa dla Niemiec) pod kątem kształtowania przyszłej polityki Niemiec i UE wobec Rosji. Wybór partii był uzależniony od ich realnych szans na wejście do Bundestagu w 2017 r. Najwięcej krytycznych opinii wobec Rosji spotkać można było w progrmach wyborczych partii liberalnych, które nawoływały do zaprzestania okupacji Krymu i wojny we wschodniej Ukrainie. Najmniej krytycznych opinii wobec Rosji znalazło się w programie partii Die Linke, która nawoływała Niemcy, UE, NATO do wyjścia naprzeciw Rosji.Wszystkie analizowane partie opowiadały się za dialogiem z Rosją. Elementy odstraszania Rosji znalazły się w programie FDP.W artykule nakreślono trzy możliwe scenariusze przyszłych relacji Niemiec z Rosją.

Słowa kluczowe: Niemcy, Rosja, Unia Europejska, partie polityczne, polityka zagraniczna 